\title{
Pancreatic lipase inhibitory activity of selected pharmaceutical agents
}

\author{
IMAD I. HAMDAN ${ }^{1 *}$ \\ VIOLET N. KASABRI ${ }^{1}$ \\ YUSUF M. AL-HIARI ${ }^{1}$ \\ DINA EL-SABAWI ${ }^{1}$ \\ HIBA ZALLOUM ${ }^{2}$ \\ ${ }^{1}$ School of Pharmacy, The University \\ of Jordan, Amman 11942, Jordan \\ ${ }^{2}$ Hamdi Mango Centre for Scientific \\ Research, The University of Jordan \\ Amman 11942, Jordan
}

Accepted September 24, 2018 Published online October 16, 2018

\begin{abstract}
Twenty-five structurally diverse compounds have been tested in vitro for their pancreatic lipase (PL) inhibitory activity. Despite the diversity of tested compounds, the relationship comprising structural attributes of the compounds could be established to correlate with the observed inhibitory activity. Compounds that exerted inhibitory action through surface activity were of different profile from the rest of compounds. When co-incubated with orlistat (OsT), important synergistic effects for some compounds (orphenadrine, gliclazide, cefuroxime and sulfacetamide) were revealed, while antagonistic effects were demonstrated for others (camphor sulfonic acid and dinitro salicylic acid). Docking studies for the most active molecules were performed and molecular interaction forces with the PL active site were identified. The results suggested co-binding of OsT along with the other inhibitor in the binding site in cases of synergistic effect but not in the case of antagonistic effect. These results were additionally supported by affinity capillary electrophoresis. In conclusion, synergistic lipase inhibitory activity between OsT and some other pharmaceutical compounds was demonstrated for the first time, which might help improve the pharmacological effect of OsT.
\end{abstract}

Keywords: pancreatic lipase, pancreatic lipase inhibitory test, pharmaceutical compounds, affinity capillary electrophoresis, docking studies

Pancreatic lipase (PL) is the main enzyme responsible for the breakdown of fatty triacylglycerol to smaller components within the digestive system to enable their absorption. Compounds that may inhibit the action of PL are expected to have favorable effects in minimizing the amount of absorbed fat and consequently a positive effect on general health (1-3). The most direct application of such compounds might be their use as antiobesity drugs, because they may limit the amount of fatty material absorbed. In spite of the great public demand for such medicines, there has been only one effective PL inhibitor available in the market - orlistat (OsT). In recent years, interest in developing more effec-

\footnotetext{
*Correspondence; email: i.hamdan@ju.edu.jo
} 
tive and safe inhibitors seems to have increased. There has been a fair number of reports that described the synthesis or isolation of new PL inhibitors, many of which exhibited only modest activity compared to OsT (4-8). PL is a rather complex enzyme whose activity is controlled by quite a number of factors such as the substrate, the product, $\mathrm{pH}$ of medium, metal ions and accessibility to the usually lipophilic substrate (1,9-11). Surfactants, represented in the body by biliary secretion, usually play a significant role in the action of PL in that they improve the interfacing between aqueous medium and essentially lipophilic substrates (11-14). Moreover, PL was shown to have a special protein moiety that functions like a lid for the binding site, which can be open upon a trigger by the presence of an oily substrate $(10,11,14)$. Co-lipase is another protein subunit necessary for optimal action of PL in the presence of bile acids, which may otherwise inhibit the lipolytic action of the enzyme (10). Such multiple factors make it difficult to precisely control or predict the activity of PL in vivo. Nevertheless, the three-dimensional structure of PL and its catalytic binding site have been characterized using X-ray crystallography when the binding site was shown to be composed of an aspartic acid-histidine-serine catalytic triad (1). In attempts to further explore the binding site of PL, twenty-five pharmaceutical compounds, most of them in clinical use, have been tested as PL inhibitors. In a previous report, the wellknown antibiotic tetracycline was found to possess an interesting alpha amylase inhibitory activity, which helped explain some of its side effects (15). Therefore, in addition to identifying new structural scaffolds for lipase inhibitors, some of the side effects of commonly used drugs might be explained by this study as well.

\section{EXPERIMENTAL}

\section{Chemicals, instruments and software}

The tested pharmaceutical compounds (Table I) were kind gifts from the Jordanian Pharmaceutical Manufacturing Company (JPM, Jordan) and Dar Al Dawa Co. Ltd. (DAD, Jordan). All tested compounds were certified to be of $>98 \%$ purity. All reagents and chemicals, including porcine pancreatic lipase PL (type II), the substrate $p$-nitrophenyl butyrate (PNPB), Tris-HCl buffer and phosphate buffer were procured from Sigma-Aldrich Co. Ltd. (UK). A UV-VIS spectrophotometer (SpectroScan 80D, Sedico Ltd, UK) was used. Affinity capillary electrophoresis (ACE) experiments were performed using an Agilent CE 1000 (Germany) instrument and electropherograms were monitored at $205 \mathrm{~nm}$. Uncoated fused silica capillaries were obtained from Composite Metal Services Ltd. (UK) and were used after being cut to proper length without further modifications.

The following software packages were utilized in the docking experiments: i) CS ChemDrawUltra 12.0.2, Cambridge Soft Corp. (http://www.cambridgesoft.com), USA; ii) OMEGA (Version2.5.1.4); iii) OpenEye Scientific Software (www. eyesopen.com), USA; iv) FRED (Version 2.2.5), OpenEye Scientific Software, (www.eyesopen.com, USA); v) BIOVIA Discovery Studio visualizer 4.5, 2015, Biovia, Accelrys Inc. (www.accelrys.com, USA).

\section{Spectrophotometric quantification of pancreatic lipase}

All tested compounds were dissolved in DMSO to prepare a stock solution (of each compound) having a concentration in the range of $2-5 \mathrm{mg} \mathrm{mL}^{-1}$. A proper aliquot of the so 
obtained solution was further diluted using $2.5 \mathrm{mmol} \mathrm{L}^{-1}$ Tris- $\mathrm{HCl}$ buffer, $\mathrm{pH} 7.4$ (containing $2.5 \mathrm{mmol} \mathrm{L}^{-1} \mathrm{NaCl}$ ) to give a series of solutions with final drug concentrations in the range of $0.2-2000 \mu \mathrm{g} \mathrm{mL} \mathrm{mL}^{-1}$, so that a dose response curve could be established. A stock solution of OsT, the reference drug, was also prepared in DMSO at a concentration of $1 \mathrm{mg}$ $\mathrm{mL}^{-1}$. Proper aliquots of the so obtained solution were further diluted in Tris- $\mathrm{HCl}$ buffer (as above) to obtain standard solutions of OsT in the range $0.0125-0.4 \mu \mathrm{g} \mathrm{mL} \mathrm{L}^{-1}$. In vitro enzymatic pancreatic lipase (PL) activity was assayed according to a previously established procedure (16). Porcine pancreatic lipase was suspended in Tris-HCl buffer (as above) to give a concentration of 200 unit $\mathrm{mL}^{-1}$. The substrate, $p$-nitrophenyl butyrate (PNPB), was dissolved in acetonitrile to obtain a solution with a concentration of $100 \mu \mathrm{mol} \mathrm{L}^{-1}$. An aliquot $(0.10 \mathrm{~mL})$ of PL solution was added to the reaction mixture. The volume was completed to $1 \mathrm{~mL}$ using Tris- $\mathrm{HCl}$ buffer before measuring the solution absorbance spectrophotometrically, at $410 \mathrm{~nm}$, and after incubation for $5 \mathrm{~min}$. The reaction, maintained at 37 ${ }^{\circ} \mathrm{C}$, was started by adding the substrate to the reaction mixture. Release of $p$-nitrophenol was measured as the increase in absorbance measured at $410 \mathrm{~nm}$ against a blank using denatured enzyme. PL was pre-incubated with each particular drug for at least $10 \mathrm{~min}$ at $37^{\circ} \mathrm{C}$ before adding the substrate. Percentage of residual activity of PL was determined for each test compound by comparing the lipase activity of PL with and without extract.

Subsequent determinations were undertaken for the tested compounds and OsT in comparison with the controls (denatured enzyme) to calculate percentage inhibition at each concentration and eventually the $50 \% \mathrm{PL}$ inhibition $\left(I C_{50}\right)$. The values are presented as mean \pm standard deviation of 3 independent experiments. Once $I C_{50}$ value for each compound was obtained, the PL inhibitory activity of each compound was also determined in the presence of OsT at a concentration level equal to the $I C_{25}$ value for each drug and OsT. The purpose of this last experiment was to get a better understanding of how the presence of more than one potential inhibitor might influence the binding to PL and the subsequent inhibitory effect, e.g., synergistic or antagonistic.

\section{Capillary electrophoresis}

In our experimental design, samples of PL were always electrophoresed in phosphate buffer ( $\mathrm{pH}$ 6.8), either alone or containing OsT or containing a test compound or containing a mixture of both OsT and the test compound. The idea behind the experimental protocol was to examine if any indication of OsT co-binding along with the other test compound could be obtained through shifts in migration times of the peaks or peak shape.

To dissolve PL for the enzyme inhibition test, a mixture was prepared of DMSO, phosphate buffer (50 mmol L-1, $\mathrm{pH} 6.8)$, methanol and calcium chloride solution $\left(0.25 \mathrm{mg} \mathrm{mL}^{-1}\right)$ in a volume ratio 1/40/10//9 $(V / V / V / V)$. PL samples were prepared by dissolving $10 \mathrm{mg}$ of the enzyme in $30 \mathrm{~mL}$ of the mixture described above. A $100-\mu \mathrm{L}$ aliquot of the so obtained solution was mixed with $200 \mu \mathrm{L}$ of methanol and vortex mixed before being injected into the affinity capillary electrophoresis (ACE) system as a PL alone sample. Samples of PL with test drug were prepared by mixing $100 \mu \mathrm{L}$ of the original PL solution with $100 \mu \mathrm{L}$ of drug solution and $100 \mu \mathrm{L}$ of methanol. Samples of a test drug with OsT were prepared by mixing 100 $\mu \mathrm{L}$ of the original PL solution, $100 \mu \mathrm{L}$ of the test drug and $100 \mu \mathrm{L}$ of OsT solution $\left(400 \mu \mathrm{g} \mathrm{mL}^{-1}\right.$ in methanol). Electrophoresis running buffer consisted of $15 \%$ acetonitrile and $16.7 \%$ methanol in $50 \mathrm{mmol} \mathrm{L}^{-1}$ phosphate buffer (pH 6.8), which was marked as blank running buffer. In 
cases where a test drug had to be added to the buffer, $1 \mathrm{~mL}$ of blank running buffer was mixed with $100 \mu \mathrm{L}$ of the test drug solution $\left(400 \mu \mathrm{g} \mathrm{mL}^{-1}\right.$ in methanol) and $100 \mu \mathrm{L}$ of methanol. When the running buffer had to contain both the test drug and OsT, $1 \mathrm{~mL}$ of blank running buffer was mixed with $100 \mu \mathrm{L}$ of the test drug solution $\left(400 \mu \mathrm{g} \mathrm{mL} \mathrm{m}^{-1}\right.$ in methanol) and $100 \mu \mathrm{L}$ of OsT solution ( $400 \mu \mathrm{g} \mathrm{m \textrm {m } ^ { - 1 }}$ in methanol). Electrophoresis of all samples was performed using uncoated fused silica capillaries cut to a length of $55 \mathrm{~cm}(75 \mathrm{~mm}$ i.d.) with an operating voltage of $15 \mathrm{kV}$. All electropherograms were monitored at $205 \mathrm{~nm}$. Samples were injected electrokinetically for $15 \mathrm{~s}$ at $18 \mathrm{kV}$. Each injection was preceded by flushing for $30 \mathrm{~s}$ with $0.1 \mathrm{~mol} \mathrm{~L}^{-1} \mathrm{NaOH}$ for $30 \mathrm{~s}$ with water and finally 2 min running buffer fill.

\section{Docking experiments}

The docking study was conducted utilizing a docking engine FRED (FRED 2009). FRED docks molecules employing a comprehensive search algorithm where it systematically searches rotations and translations of each conformer of the ligand within the active site and filters unrealistic poses. Pose generation is followed by estimating the strength of ligand-target interactions (scoring) to guide conformational sampling and estimating the biological activity. Final docked conformations (poses) are ranked and selected according to their scores. For PL, we had previously identified the optimal docking configuration and scoring function by the self-docking of the co-crystallized ligand. These parameters were further validated experimentally when they were used to estimate the bioactivity of some natural and synthetic PL inhibitors and were predicted by simulated docking to bind within the PL active site.

The 2D chemical structures of docked compounds (Fig. 2) were sketched in Chemdraw Ultra (12.0.2) and saved in the MDL molfile format. Subsequently, a group of energetically accessible conformers was generated using OMEGA software (OMEGA, 2013; Version2.5.1.4), OpenEye Scientific Software (www. eyesopen.com, USA). OMEGA rapidly generates conformational ensembles of small molecules using a fragment-based library in order to build initial models of structures by assembling these fragment templates followed by a rule-based torsion search stage. The generated conformers were saved in the SD format. The 3D geometry of pancreatic lipase (PL) was retrieved from the Protein Data Bank (PDB code: 1LPB, resolution $2.46 \AA$ ) (10). Hydrogen atoms were added to the protein using the BIOVIA discovery studio visualizer templates (Discovery Studio visualizer 4.5, 2015 Biovia, Accelrys Inc. (www.accelrys.com,USA) for protein residues. No energy minimization for the protein structure was done. The docking study was conducted in the presence of explicit water molecules. Test compounds (Fig. 2) were docked into the binding pocket of PL using the FRED (FRED (Version 2.2.5), OpenEye Scientific Software, (www. eyesopen.com, USA) software. Ligand conformers and protein structure are treated as rigid entities during the docking process. FRED docking roughly consists of 2 steps: shape fitting and optimization. In the fitting phase, the ligand is placed into a grid box including all active-site atoms, then a series of three optimization filters are employed (optimization phase). The filters include rigid body optimization, refining the position of the ligand hydroxyl hydrogen atoms and finally optimization of the ligand pose in the dihedral angle space (FRED 2009; FRED (Version 2.2.5), OpenEye Scientific Software, (www.eyesopen. com, USA). Generated conformers of the test compounds were used as input to the FRED software. Docking settings that succeeded in reproducing the experimental pose of the co-crystallized ligand (MUP901, C11P) were employed (10). Our previously optimized FRED docking simulation parameters for pancreatic lipase have been reported (17). 


\section{RESULTS AND DISCUSSION}

\section{Lipase inhibitory activity}

The obtained $I C_{50}$ value for OsT was $0.2 \pm 0.01 \mu \mathrm{mol} \mathrm{L}{ }^{-1}$, which is comparable to $I C_{50}$ values reported elsewhere (18), thus promoting the validity and reliability of the recruited PL activity assay. Comparable to OsT performance, a marked concentration dependent PL inhibition trend was obtained for various test drugs. The obtained $I C_{50}$ values for the tested compounds are presented in Table I. While 8 of the 25 tested compounds were shown to have reasonable lipase inhibitory activity, 7 had no detectable activity under the employed assay conditions, which served as a negative control that helped in validating our results.

Some of the tested compounds were shown for the first time to have reasonable PL inhibitory activities, e.g., orphenadrine (OrP) and gliclazide (GzD). The most active compounds exhibited $I C_{50}$ values in the range of $20-50 \mu \mathrm{mol} \mathrm{L}-1$, which obviously could not mount to the activity of the standard inhibitor OsT, but is quite comparable to the range of $I C_{50}$ values reported for naturally occurring and synthetic lipase inhibitors (3). The list of most active compounds with $I C_{50}<50 \mu \mathrm{mol} \mathrm{L}-1$ included acetylsalicylic acid (AsA), camphor sulfonic acid (CsA), dinitro salicylic acid (DnS), gamma-anilino naphthalene sulfonic acid (GsA), gliclazide (GzD), orphenadrine (OrP), salicylic acid (ScA) and sulfacetamide (SfD). From the above mentioned compounds, only ScA had been previously reported to have a lipase inhibitory activity, which was previously investigated as lipase inhibitor along with other phenolic acids (19). One report has shown the ability of acetylsalicylic acid to improve the lipid profile for high fat-fed rats but attributed this effect to the influence on liver enzymes (20).

Therefore, we herein provide direct evidence that the effect of AsA might be, at least partly, mediated by its ability to inhibit PL. DnS was the most active compound with $I C_{50}$ value of $20.4 \mu \mathrm{mol} \mathrm{L}{ }^{-1}$. In accordance with the previous reports and consequent predictions, it was logical to observe lipase inhibitory activity for simple aromatic acids: DnS,

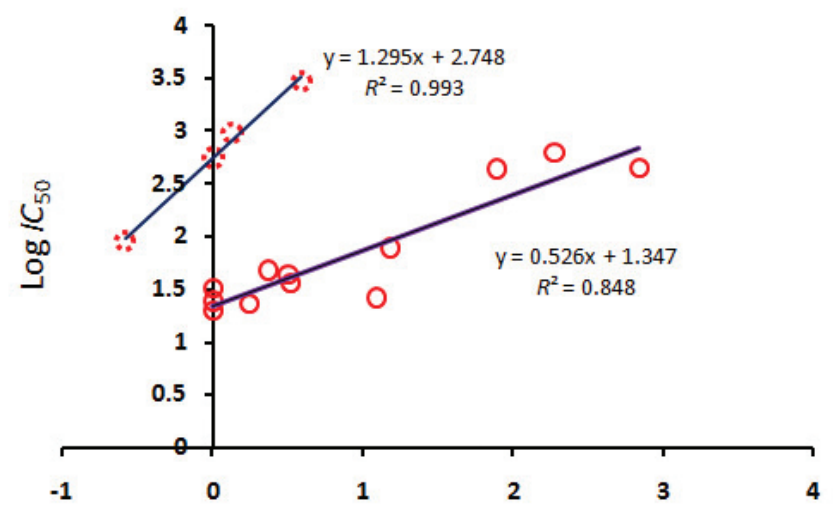

\section{Activity predictor value $A$}

Fig. 1. Plot of $\log I C_{50}$ against the activity predictor value $(A)$. 
<smiles>O=C(NN1CC2CCCC2C1)NS(=O)(=O)c1ccccc1</smiles>

GzD<smiles>O=S(=O)(O)c1cccc2cccc(Nc3ccccc3)c12</smiles>

GsA<smiles>CC1(C)C2CCC1(CS(=O)(=O)O)C(=O)C2</smiles>

CsA<smiles>Cc1cccc(C(OCCN(C)C)c2ccccc2)c1</smiles>

OrP

Dns<smiles>CC(=O)Oc1ccccc1C(=O)O</smiles>

AsA<smiles>CC(=O)NS(=O)(=O)c1ccc(N)cc1</smiles>

SfD

Fig. 2. Chemical structures of the seven most active compounds: gliclazide (GzD), gamma-anilino sulfonic acid (GsA), camphor sulfonic acid (CsA), orphinadrine (OrP), dinitrosalicylic acid (DnS), acetyl salicylic acid (AsA) and sulfacetamide (SfD).

AsA and ScA $(19,21)$. Most interesting perhaps was the PL inhibitory activity of the sulfonylurea drug GzD, the sulfonamide SfD and the basic drug OrP, which had not been previously reported to have such an activity. Short term use of GzD by diabetic patients was shown to improve postprandial lipemia (22), which might support our findings. More relevant, perhaps, were some recent reports which demonstrated the inhibition of the related enzyme diacylglycerol lipase by glycine sulfonamide derivatives that can be structurally related to $\mathrm{GzD}$ and $\operatorname{SfD}(23,24)$. Thus, our findings support the potential importance of sulfonamides or sulfonylurea derivatives as inhibitors of PL. OrP, a basic compound, represents a structurally unusual lipase inhibitor as many of the reported inhibitors were based on carboxylic acid or phenol function $(5,7,19,25)$. It was interesting to observe the lower activity of surfactants like sodium dodecyl sulfate (SdS) and sodium dioctylsulfonate (DoS). Surfactants have been reported to have a complex effect on lipase activity as they affect various equilibria that are relevant to the lipolytic activity of the enzyme (12). For example, their attachment to the surface of the oily substrate through their lipophilic tails forms a shield that hinders the access of PL to the oil/water interface. On the other hand, surfactants increase the solubility of the substrate, which may enhance the lipolytic action of the enzyme.

\section{Structure-activity studies}

Another interesting inhibitory compound was aspartic acid (AsP). Although AsP did not exhibit particularly high inhibitory activity $\left(I C_{50}=91.7 \mu \mathrm{mol} \mathrm{mL}^{-1}\right)$, it had a slightly polar structure, which is rather unusual for a lipase inhibitor. Therefore, attempts were made to better understand the correlation between structural features and PL inhibitory activity. Several algorithms were randomly generated in an effort to find a possible correlation with the observed lipase inhibitory activities, using molecular descriptors such as log $P, \mathrm{p} K_{\mathrm{a}}$, number of hydrogen bonds acceptors, number of hydrogen bonds donors, rotatable bond count and polar surface area. Eventually, an algorithm that reasonably correlates the 
observed in vitro inhibitory effect of a compound with its molecular descriptors was obtained, and is given by the equation:

$$
A=\left[\left(\log P+\mathrm{p} K_{\mathrm{a}}\right) /(P+\mathrm{Hd}+\mathrm{Ha})\right] \times[(\mathrm{Rb} \times \mathrm{PSA}) / \mathrm{com}]
$$

where $A$ is the estimated activity predictor, $P$ is the partition coefficient, $\mathrm{Hd}$ is the number of hydrogen bond donors, $\mathrm{Ha}$ is the number of hydrogen bond acceptors, $\mathrm{Rb}$ is the number of rotatable bonds, PSA is the polar surface area and com is complexity. It is interesting that, when $\log I C_{50}$ was plotted against the A value, and excluding inactive compounds, cefuroxime axetil (CfA) and uric acid (UrA) (Table I), two clear linear relationships could be distinguished (Fig. 1). Seemingly, one of them reflected the lower activity group of compounds while the other reflected more active compounds, but both exhibited good determination coefficients of 0.85 and 0.99 , respectively. Thus, we proposed that the tested group of compounds bind in two different modes to the enzyme, leading to its inhibition. Consequently, two distinct linear relationships were observed. Interestingly, the low activity group of inhibitors included compounds such as AsP and diethylbarbituric acid (DbS) (Table I) that do not possess the typical features of PL inhibitors, e.g., phenol or carboxylic acid. On the other hand, the more active group included compounds with typical structural requirements for PL inhibitory activity, e.g., ScA, AsA in addition to new structural nuclei reported in this study only, i.e., OrP, GzD and SfD.

In order to gain a better insight into the binding modes of the examined inhibitors, two studies were conducted. In one, the inhibitory activity was assessed for each compound in the presence of the standard inhibitor OsT, both at $I C_{25}$. The reason was that, if there was no interaction between the binding process/mode of OsT and that of the inhibitor being tested, then the observed inhibition percentage would consistently be $\sim 50 \%$. Percentages higher than $50 \%$ would indicate synergism in the action of the two drugs and most probably different interaction/binding modes with the active site of the enzyme. Some of the tested compounds exhibited a negative value of inhibition when tested in the presence of OsT, thus suggesting some degree of enzymatic activation rather than inhibition, with DoS having the most obvious effect $(-16 \%)$. Careful examination of these compounds revealed that they all shared some degree of surface activity as they are composed of a polar ionized part (sulfonic or carboxylic) and another lipophilic part. Therefore, the observed enzyme activation when combined with OsT could be understood as surfactants increasing the effective concentrations of both the enzyme and the substrate (12-14). Being a highly lipophilic molecule with a positively charged polar head, OsT was potentially inactivated in the presence of the negatively charged micelle molecules by being impeded within the micellar structure, i.e., forming mixed micelles as previously reported with bile salts (11). A few other compounds, e.g., DnS and cephalexin (CpL), demonstrated low positive values $(0-20 \%)$ of inhibition, which indicates antagonistic rather than synergistic action with OsT. The result for DnS was particularly interesting because it exhibited the lowest $I C_{50}$ in the absence of OsT but demonstrated obvious antagonism in its presence.

Compounds that exhibited an inhibition effect within $\pm 20 \%$ of the expected value of $50 \%$, (e.g., AsA, AvS and ScA) were considered to behave according to expectations, i.e., they simply showed an additive effect with OsT, suggesting that they may bind to the enzyme in a similar manner as to OsT. On the other hand, a fair number of compounds (GzD, OrP, UrA, AsA, MfA, DbS, SfD and CfA) appeared to have a synergistic effect with OsT, with obtained activities $>80 \%$ and in some cases $>90 \%$. It is noteworthy that even compounds with very 


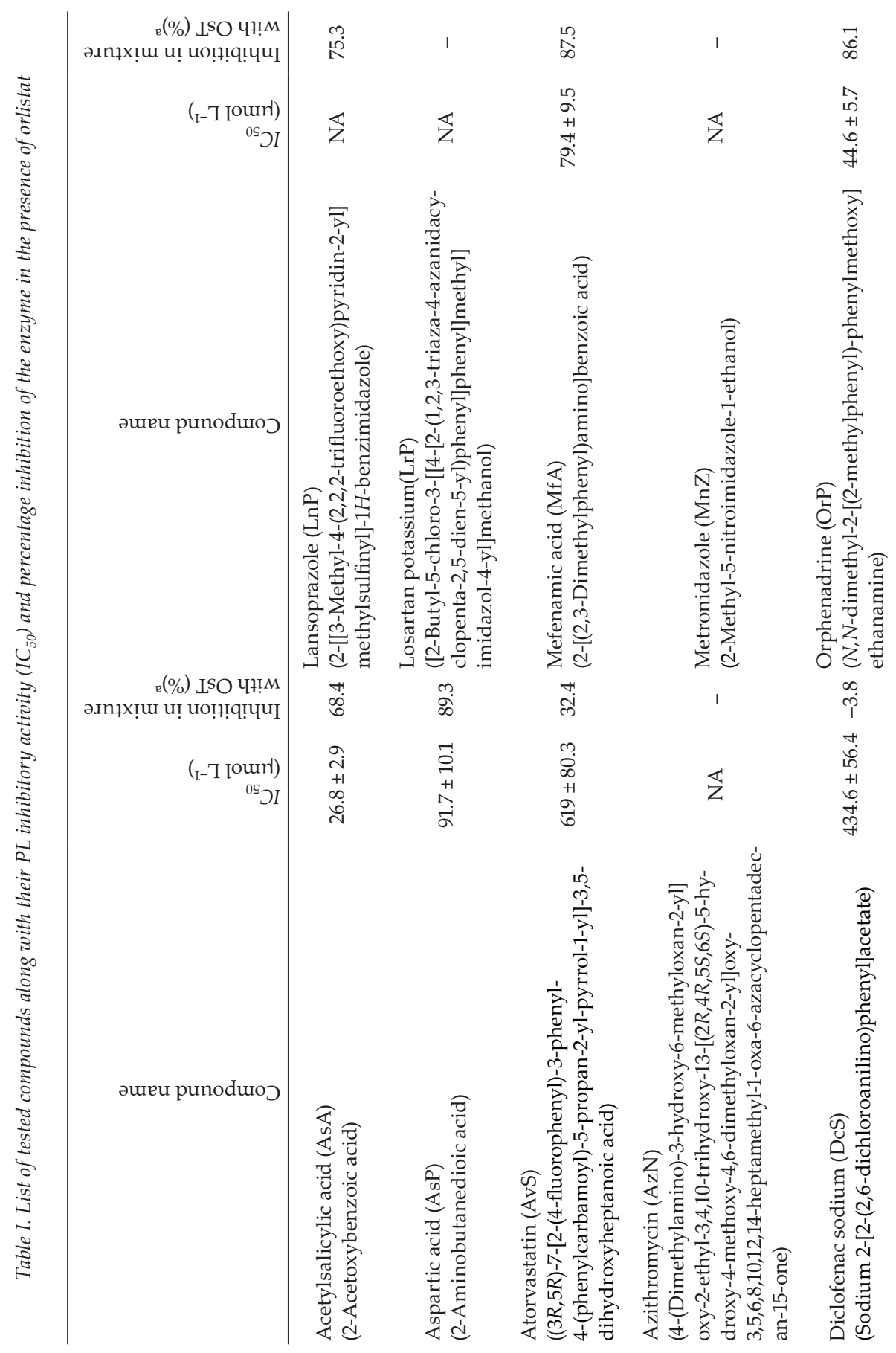




\begin{tabular}{|c|c|c|c|c|c|}
\hline 1 & 1 & ?ु & $\stackrel{0}{+}$ & $\begin{array}{l}\infty \\
\stackrel{\infty}{0} \\
\end{array}$ & म̆ \\
\hline 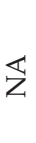 & $\overleftrightarrow{Z}$ & $\begin{array}{l}n \\
+ \\
+1 \\
\dot{+} \\
\dot{0} \\
0\end{array}$ & $\begin{array}{l}n \\
\infty \\
10 \\
+1 \\
\infty \\
\infty \\
\infty \\
+\end{array}$ & $\begin{array}{l}n \\
\hat{b} \\
+1 \\
a \\
0 \\
0 \\
0\end{array}$ & $\begin{array}{l}\stackrel{a}{N} \\
+1 \\
\sim \\
\tilde{N}\end{array}$ \\
\hline
\end{tabular}

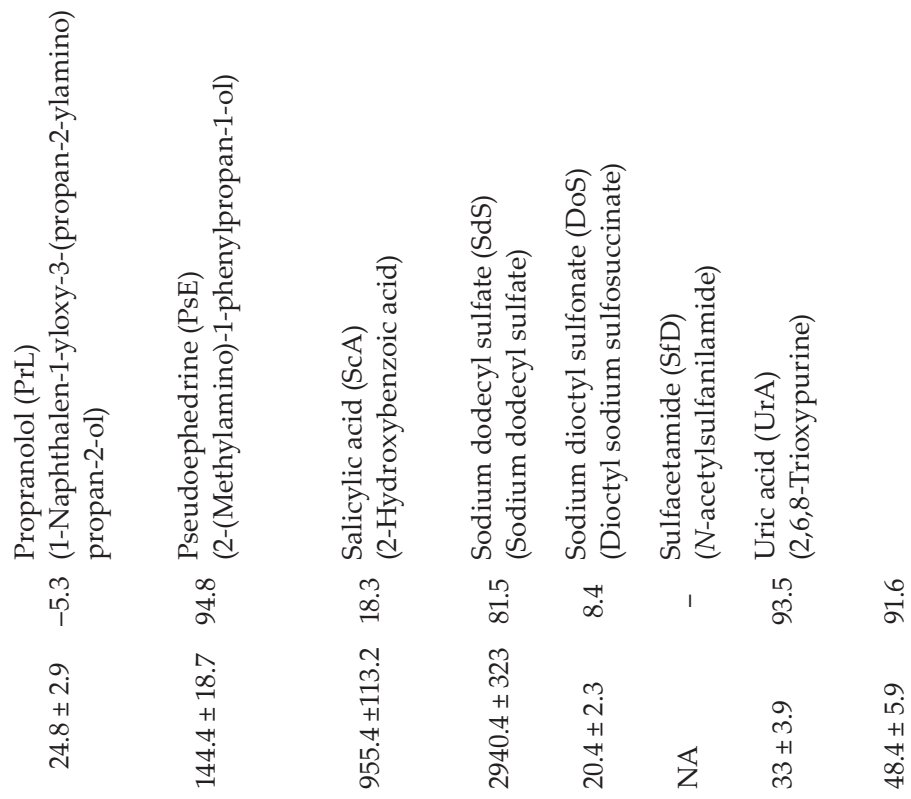

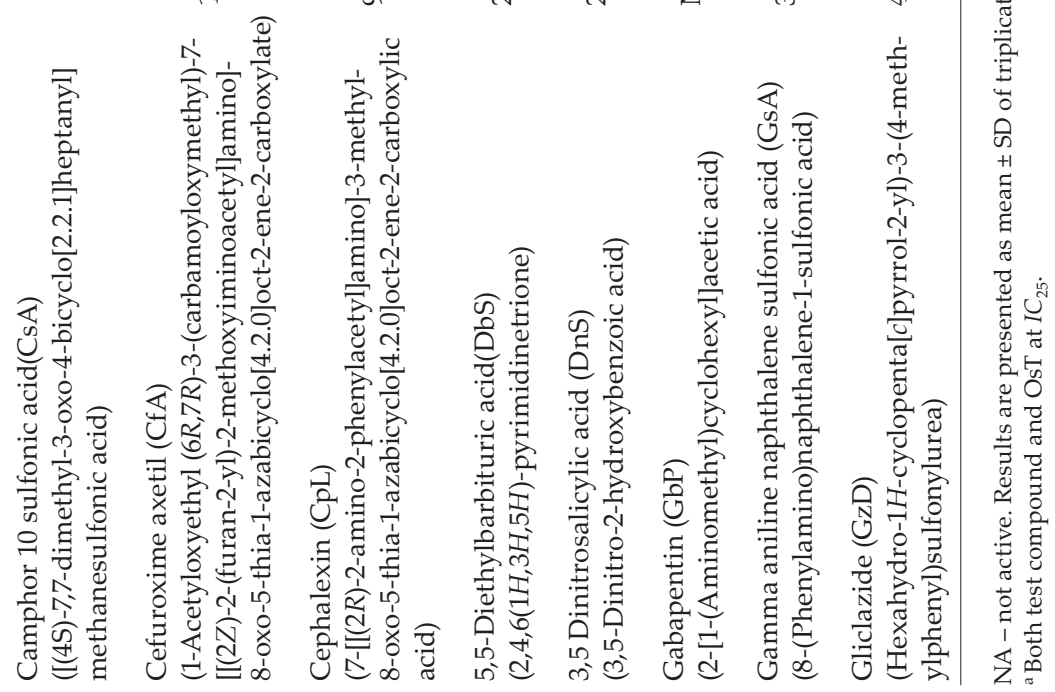


low activities on their own, like DnS and CfA, were shown to substantially enhance the OsT activity. These observations suggest that the tested compounds with synergistic effects bind to the enzyme in different modes with consequent closure of some parts of the active site, but exposure of OsT binding site and enhanced activity. On the other hand, the binding of compounds (with antagonistic activity) may result in obstruction of the OsT binding site; therefore, they may exhibit a reasonable inhibitory activity when present alone but the overall activity would be diminished when present together with OsT. Our findings could help explain potential drug-drug interaction side effects through their synergistic or antagonistic action with OsT. Attempts were, however, made to further explore the mechanism by which different inhibitors interact with the enzyme.

\section{Docking studies}

Simulated molecular docking is basically a conformational sampling procedure in which various docked poses/conformations are explored to identify the correct one $(26,27)$. Therefore, the seven most active test compounds (Fig. 2) were docked into the binding pocket of PL with the binding site being defined from the crystallographic structure of a bound high-affinity ligand (MUP901, C11P). Fig. 3 shows the highest ranking pose for the most active compounds when docked within the binding site of PL along with the scoring function calculated using Chemgauss according to their binding affinities. Some similarities were noticed in the binding profiles between the self-docked ligand (MUP901, C11P) and some of the tested drugs. GzD, which exhibited the highest estimated affinity, fitted nicely within the PL binding pocket. The benzene ring of GzD undergoes many hydrophobic interactions of aromatic $\pi-\pi$ (pi-pi) stacking and $\pi$-alkyl with several key amino acid residues, including Phe-77, Phe-215, Tyr-114 and Pro-180, which collectively form a lipophilic pocket within the binding pocket of PL. Other hydrophobic attractions occurred with His-263 and Leu-264 amino acid residues, in addition to two hydrogen bonds formed with Phe-77 amino acid which stabilized the ligand-protein complex, as shown in Fig. 3a. GsA was ranked second to GzD and also underwent aromatic $\pi-\pi$ stacking between its naphthalene ring and Tyr-114 residue in addition to $\pi$-alkyl with Pro-180. Similarly to GzD, hydrophobic attraction occurred with His-263 amino acid residue (Fig. 3b). It is, however, noteworthy that both of these compounds (GzD and GsA) missed interaction with Ser-152, which had previously been shown to be very significant for inhibition to take place (28). This might account for the observation that despite their exhibiting the highest Chemgauss score rank, they were not the most active inhibitors according to the in vitro lipase inhibitory test results. The importance of interaction with the Ser-152 residue was reinforced with the binding of DnS, where it appeared to form two strong hydrogen bonds with Ser-152 and another weaker with Pro180 , in addition to two hydrophobic $\pi-\pi$ stacking interactions between its aromatic ring and both of Phe-215 and Tyr-114 amino acid residues. Experimentally, DnS exhibited the best inhibitory activity among all the tested compounds, which is in accord with its observed high degree of interaction with the active site including Ser-152. However, DnS and CsA were ranked next to GzD and GsA according to the Chemgauss score. CsA also formed many hydrophobic $\pi$-alkyl interactions with the hydrophobic side and the aromatic rings of Phe-77, Phe-215 and Tyr-114 amino acid residues, and three hydrogen bonds with Ser-152, His-263 and Phe-77, which appeared to stabilize the ligand-protein complex, thus explaining the observed high activity of CsA. On the other hand, OrP underwent only hydrophobic stacking interactions (aromatic $\pi-\pi$ stacking and $\pi$-alkyl stacking) between its benzene/ 
benzyl rings and Phe-215, Tyr-114, His-263, Pro-180 and Ala-178 amino acid residues of PL and was thus in accord with the observed relatively higher $I C_{50}$ for OrP.

AsA and SfD demonstrated a considerable number of tight interactions with the enzyme binding site, in accordance with their observed lowest $I C_{50}$ value range. AsA formed three hydrogen bonds with Ser-152, Tyr-114 and a weaker one with Pro-180 amino acids, but it only formed one hydrophobic aromatic $\pi-\pi$ stacking with Tyr-114 residue. With SfD, the amine group of the aniline ring formed two strong hydrogen bonds with Ser-152 and Phe-215 while its aromatic ring underwent three hydrophobic interactions of aromatic $\pi-\pi$ stacking and $\pi$-alkyl with Tyr-114, Pro-180 and Ala-178. Although no exact quantitative relationship was obtained from docking studies to describe the in vitro inhibitory activity, they provided some semi-quantitative explanations of the observed trends in inhibitory activities. For example, the two least active compounds were the ones shown not to be capable of hydrogen bonding with the crucial Ser-152 in the active site. On the other hand, the remaining 4 out of 7 compounds that were shown to be relatively highly active were confirmed to bind to Ser-152 through strong hydrogen bonding.

The possible explanation why DnS and CsA in particular, in the group of relatively highly active compounds, led to antagonism rather than synergism with OsT, and according to the results provided by docking experiments, we may conclude the following: if the ligand is bulky enough to physically obstruct the binding site, and additionally tightly attach through Ser residue in particular, then there would be a minimal chance for OsT to reach the binding site and consequently its inhibitory activity would be antagonized (as with DnS and CsA; Fig. 3). On the other hand, if it cannot bind to Ser residue (even if it is bulky), or if it is not bulky enough, then there would be a space for OsT to reach the active site and accomplish its inhibitory action, which might be even potentiated by the additional binding of the test ligand (Fig. 3). In search for further support, simple experiments employing affinity capillary electrophoresis were performed.

\section{Affinity capillary electrophoresis}

Affinity capillary electrophoresis has been widely used in the studies of ligand-protein interactions (29). The obtained electropherograms (e-grams) showed that the presence of OsT in the running buffer caused an obvious shift in migration time $\left(t_{\mathrm{m}}\right)$ and the peak shape change of PL (Fig. 4), thus pointing to the possible binding to the enzyme. However, the direction of change in $t_{\mathrm{m}}$ (increase from 5.6 to $6.5 \mathrm{~min}$ ) was rather unexpected because OsT exists as a positively charged molecule under the employed conditions, and its binding to the enzyme would impart a more positive charge, leading to faster mobility to the cathode (detector end) and thus lower $t_{\mathrm{m}}$.

Hence, it appears that binding of OsT to the enzyme induces some conformational changes in the enzyme three-dimensional structure that favors mobility in the opposite direction (anode). As electroosmotic flow (EOF) is larger than the intrinsic mobility of PL, it manages to reverse the direction of migration of the enzyme, but after an additional delay in $t_{\mathrm{m}}$. These results are in accord with previous reports that confirmed the occurrence of inhibitor-induced structural changes that were mainly opening of the lid loop, making it closer to the co-lipase part potentiating its association with PL, i.e., creating a more condensed structure (30). When incorporated in the running buffer, all other highly active test compounds $\left(I C_{50}<50 \mu \mathrm{mol} \mathrm{L}^{-1}\right)$ resulted in a similar kind of shift in the migra- 

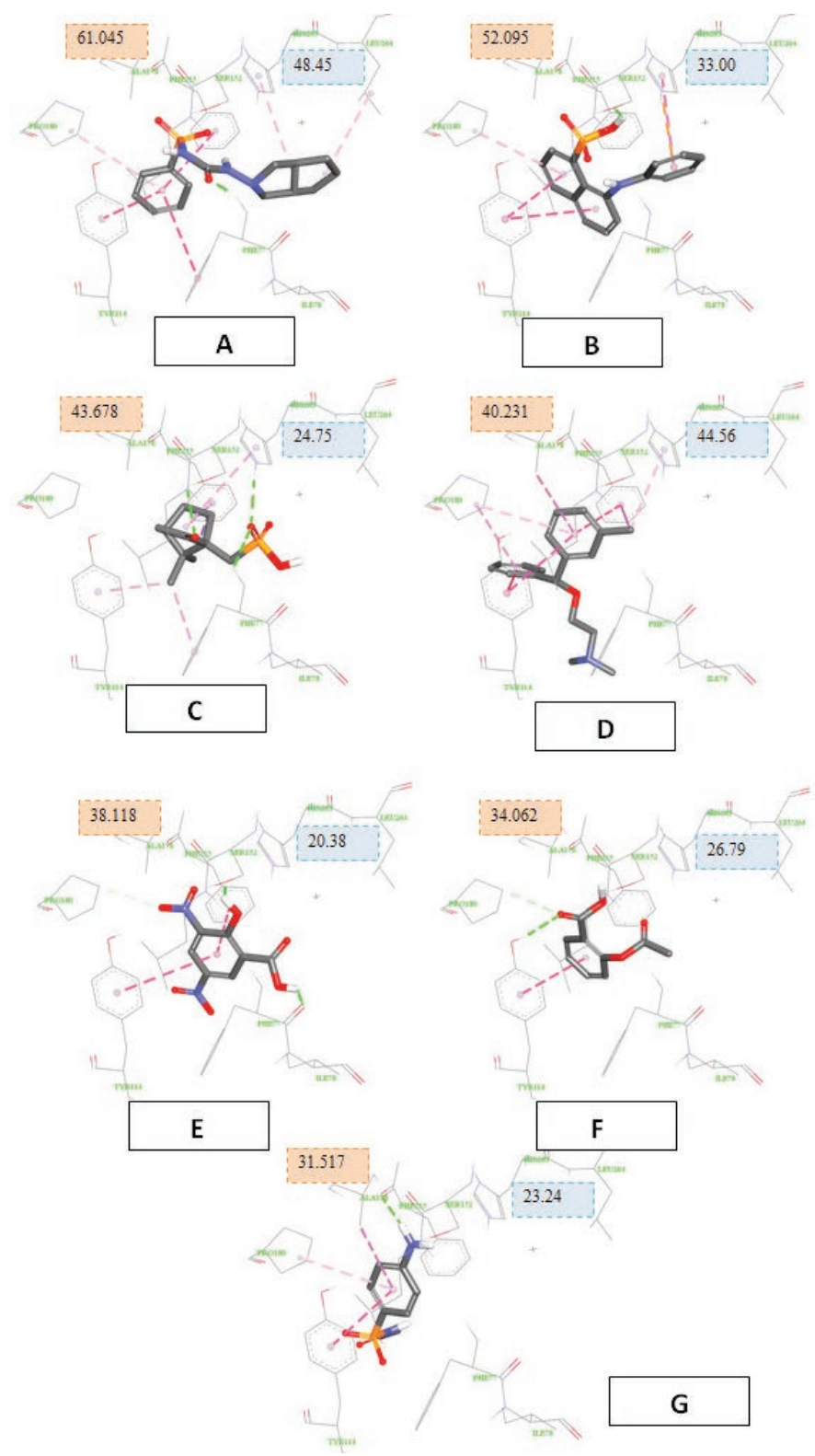

Fig. 3. Detailed view of the docked pose of tested drugs and the corresponding interacting amino acid moieties within the binding site of pancreatic lipase enzyme (code: $A=G z D, B=G s A, C=C s A, D=$ OrP, $\mathrm{E}=\mathrm{DnS}, \mathrm{F}=\mathrm{AsA}$ and $\mathrm{G}=\mathrm{SfD}) . \mathrm{IC}_{50}$ value and estimated Chemguass score are shown on the right and left of each compound, resp.

Key to net version: green $\mathrm{H}$ - bonding; pale green weaker H-bonding; pink, pale pink and orange are all hydrophobic interactions. 
tion time of PL (regardless of their charge) although to various degrees. Though small, the observed shift is most likely a result of the conformational changes induced by the inhibitor binding rather than a non-specific effect caused by the incorporation of the drug in the running buffer. Moreover, when both the test drug and OsT were incorporated in the running buffer, the change in $t_{\mathrm{m}}$ and peak shape was larger than that observed in the case of the test drug or OsT alone, except for DnS. Namely, addition of OsT to the running buffer containing DnS did not bring about any further change in the $t_{\mathrm{m}}$ of PL. For other test drugs, including OrP, SfD and GzD, addition of OsT to the running buffer containing the test drug was shown to result in an additional change in $t_{\mathrm{m}}$ (Fig. 4). The obtained experimental data are in accord with the hypotheses proposed above, where OsT could bind to PL along with other test drugs but not with DnS, with consequent observed synergism with other test compounds but not with DnS. Our findings are the first to suggest a potential synergistic inhibitory effect of two PL binding agents (inhibitors). Along this line, we propose further optimization of the test drugs using the highly promising fragment based drug design approach that has been shown successful in a number of cases, particularly when two molecular fragments show a synergistic effect $(31,32)$. Fragment based drug optimization suits our test compounds in particular because many of them showed modest inhibitory activity that was synergistically enhanced with the standard PL inhibitor OsT.

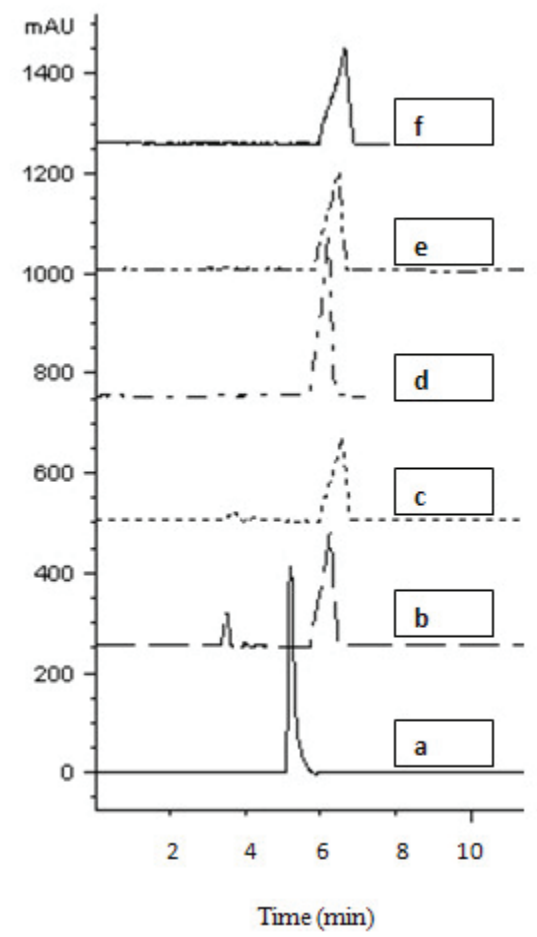

Fig. 4. Representative electropherograms of PL in phosphate buffer ( $\mathrm{pH}$ 6.8): a) alone, b) with both OrP and OsT, c) with OrP, d) with OsT, e) with DnS, f) with both DnS and OsT. Note the change of $t_{\mathrm{m}}$ of PL from $5.6 \mathrm{~min}$ (in a) to $6.5 \mathrm{~min}$ (in e and f). 


\section{CONCLUSIONS}

A considerable number of commonly used pharmaceutical compounds were shown to have significant PL inhibitory activity. The most effective PL inhibitory compounds included dinitro salicylic acid (DnS), camphor sulphonic acid (CsA), gliclazide (GzD), gama-anilino sulfonic acid (GsA) and orphenadrine (OrP). An algorithm that reasonably described the correlation between the observed inhibitory activity and structural parameters could be found. According to the correlation equation comprising $\mathrm{pKa}, \log P$, molecular surface area and molecular complexity, two linear relationships could be distinguished reflecting the two major modes of inhibitions caused by two groups of compounds. While one of the mechanisms was through the usual inhibition by binding to the enzyme active site, the other that described the activity of the lower activity compounds appeared to be achieved at least in part through surface activity. Inhibitory activity test in the presence of both the test drug and OsT revealed synergistic and antagonistic action of various tested drugs with OsT. Most interesting perhaps was the observation that some compounds with little activity demonstrated a significant synergistic effect with OsT. On the other hand, DnS, the compound with the lowest $I C_{50}$ value, appeared to have some antagonistic effect with OsT rather than synergism.

Docking studies revealed the ability of test compounds to bind to the active site in a similar manner to that of the well-known standard inhibitors. While GzD and GsA did not show significant binding to the amino acid having the decisive role in the inhibitory effect of PL (Ser-152), they showed strong interactions through different residues that provided the highest Chemgauss scores as calculated by the software. Therefore, molecules which did not interact with Ser-152, the most important residue in PL, exhibited the lowest in vitro inhibitory activity among the list of highest ranking active compounds. In addition, such compounds appeared to allow for co-binding of OsT to the binding site, resulting in enhanced inhibitory activity. Other compounds which exhibited ability to strongly interact with the binding site (Ser-152 in particular) did not seem to allow for co-binding of OsT and thus they had an antagonistic rather than synergistic effect. Further studies are needed, perhaps using crystallography, to further confirm the occurrence of co-binding to the enzyme. We highly recommend the use of the compounds investigated in this study in fragment-based drug design to build up and potentially reach new ceilings of PL inhibitory activities.

Abbreviations, acronyms, symbols. - ACE - affinity capillary electrophoresis, AsA - acetylsalicylic acid, AsP - aspartic acid, AvS - atorvastatin, AzN - azithromycin, CfA - cefuroxime axetil, CpL cephalexin, CsA - camphor sulfonic acid, DbS - diethylbarbituric acid, DcS - diclofenac sodium, DMSO - dimethyl suloxide, DnS - dinitro salicylic acid, DoS - sodium dioctylsulfonate, e-gram electropherogram, EOF - electroosmotic flow, GbP - gabapentin, GsA - gamma-anilino naphthalene sulfonic acid, GzD - gliclazide, LnP - lansoprazole, LrP - losartan potassium, MfA - mefenamic acid, MnZ - metronidazole, OrP - orphenadrine, OsT - orlistat, PL - pancreatic lipase, PNPB - $p$-nitrophenyl butyrate, PrL - propranolol, PsE - pseudoephedrine, ScA - salicylic acid, SdS - sodium dodecyl sulfate, SfD - sulfacetamide, UrA - uric acid.

Acknowledgements. - The authors thank the Deanship of Academic Research at the The University of Jordan for funding this work [1630 and 1745] and for continuous support. We are also grateful for the Scientific Research Fund - The Ministry of Higher Education [MPH/1/05/2014]. The authors also thank the OpenEye Scientific Software Corporation for providing a free FRED software license. 


\section{REFERENCES}

1. F. K. Winkler, Structure of human pancreatic lipase, Nature 343 (1990) 771-774; https://doi. org/10.1038/343771a0

2. S. Ransac, Y. Gargouri, F. Marguet, G. Buono, C. Beglinger, P. Hildebrand, H. Lengsfeld, P. Hadváry and R. Verger, Covalent inactivation of lipases, Methods Enzymol. 286 (1997) 190-231; https:// doi.org/10.1016/S0076-6879(97)86012-0

3. G. Singh, S. Suresh, B. V. Krishna and K. R. Kumar, Lipase inhibitors from plants and their medical applications, Int. J. Pharm. Pharm. Sci.7 (2015) 1-5.

4. E. Kato, M. Yama, R. Nakagomi, T. Shibata, K. Hosokawa and J. Kawabata, Substrate-like water soluble lipase inhibitors from Filipendula kamtschatica, Bioorg. Med. Chem. Lett. 22 (2012) 6410-6412; https://doi.org/10.1016/j.bmcl.2012.08.055

5. Y. Narita, K. Iwai, T. Fukunaga and O. Nakagiri, Inhibitory activity of chlorogenic acids in decaffeinated green coffee beans against porcine pancreas lipase and effect of a decaffeinated green coffee bean extract on an emulsion of olive oil, Biosci. Biotechnol. Biochem. 76 (2012) 2329-2331; https://doi.org/10.1271/bbb.120518

6. E. Mentese, F. Yılmaz, N. Karaali, S. Ülker and B. Kahveci, Rapid synthesis and lipase inhibition activity of some new benzimidazole and perimidine derivatives, Bioorg. Khim. 40 (2014) 363-369; https://doi.org/10.1134/S1068162014030091

7. Y. H. Jo, S. B. Kim, Q. Liu, J. W. Lee, B. Y. Hwang and M. K. Lee, Benzylated and prenylated flavonoids from the root barks of Cudrania tricuspidata with pancreatic lipase inhibitory activity, Bioorg. Med. Chem. Lett. 25 (2015) 3455-3457; https://doi.org/10.1016/j.bmcl.2015.07.017

8. S. N. Sridhar, G. Ginson, P. O. Venkataramana Reddy, M. P. Tantak, D. Kumar and A. T. Paul, Synthesis, evaluation and molecular modeling studies of 2-(carbazol-3-yl)-2-oxoacetamide analogues as a new class of potential pancreatic lipase inhibitors, Bioorg. Med. Chem. 25 (2017) 609620; https://doi.org/10.1016/j.bmc.2016.11.031

9. A. M. Brzozowski, U. Derewenda, Z. S. Derewenda, G. G. Dodson, D. M. Lawson, J. P. Turkenburg, F. Bjorkling, B. Huge-Jensen, S. A. Patkar and L. Thim, A model for interfacial activation in lipases from the structure of a fungal lipase-inhibitor complex, Nature 351 (1991) 491-494; https:// doi.org/10.1038/351491a0

10. M. P. Egloff, L. Sarda, R. Verger, C. Cambillau and H. van Tilbeurgh, Crystallographic study of the structure of colipase and of the interaction with pancreatic lipase, Protein Sci. 4 (1995) 44-57; https://doi.org/10.1002/pro.5560040107

11. A. Bourbon-Freie, R. E. Dub, X. Xiao and M. E. Lowe, Trp-107 and trp-253 account for the increased steady state fluorescence that accompanies the conformational change in human pancreatic triglyceride lipase induced by tetrahydrolipstatin and bile salt, J. Biol. Chem. 284 (2009) 14157-14164; https://doi.org/10.1074/jbc.M901154200

12. V. Delorme, R. Dhouib, S. Canaan, F. Fotiadu, F. Carrièreand and J. F. Cavalier, Effects of surfactants on lipase structure, activity, and inhibition, Pharm. Res. 8 (2011) 1831-1842; https://doi. org/10.1007/s11095-010-0362-9

13. P. Alam, G. Rabbani, G. Badr, B. M. Badr and R. H. Khan, The surfactant-induced conformational and activity alterations in Rhizopus niveus lipase, Cell Biochem. Biophys. 71 (2015) 1199-1206; https:// doi.org/10.1007/s12013-014-0329-2

14. E. Mateos-Diaz, S. Amara, A. Roussel, S. Longhi, C. Cambillau and F. Carrière, Probing conformational changes and interfacial recognition site of lipases with surfactants and inhibitors, Methods Enzymol. 583 (2017) 279-307; https://doi.org/10.1016/bs.mie.2016.09.040

15. I. I. Hamdan, F. Afifi and M. O. Taha, In vitro alpha amylase inhibitory effect of some clinicallyused drugs, Pharmazie 59 (2004) 799-801. 
16. Y. Bustanji, M. Mohammad Mohammad, M. Hudaib, K. Tawaha, I. M. Al-Masri, H. S. Al Khatib, A. Issa and F. Q. Alali, Screening of some medicinal plants for their pancreatic lipase inhibitory potential, Jordan J. Pharm. Sci. 4 (2011) 81-88.

17. FRED (version 2.2.5) 2009. OpenEye Scientific Software (www.eyesopen.com), Santa Fe, USA.

18. S. Habtemariam, The anti-obesity potential of sigmoidin A, Pharm. Biol. 50 (2012) 1519-1522; https://doi.org/10.3109/13880209.2012.688838

19. M. Karamać and R. Amarowicz, Inhibition of pancreatic lipase by phenolic acids-examination in vitro, Z. Naturforsch. C. 51 (1996) 903-905.

20. J. A. van Diepen, I. O. C. M. Vroegrijk, J. F. P. Berbée, S. E. Shoelson, J. A. Romijn, L. M. Havekes, P. C. N. Rensen and P. J. Voshol, Aspirin reduces hypertriglyceridemia by lowering VLDL-triglyceride production in mice fed a high-fat diet, Am. J. Physiol. Endocrinol. Metab. 301 (2011) 1099-1107; https://doi.org/10.1152/ajpendo.00185.2011

21. A. Kumarand and S. Chauhan, Monte Carlo method based QSAR modeling of natural lipase inhibitors using hybrid optimal descriptors, SAR QSAR Environ. Res. 28 (2017) 179-197; https://doi. org/10.1080/1062936X.2017.1293729

22. R. Emral, O. Köseoğlulari, V. Tonyukuk, A. R. Uysal, N. Kamel and D. Corapcioğlu, The effect of short-term glycemic regulation with gliclazide and metformin on postprandial lipemia, Exp. Clin. Endocrinol. Diabetes 113 (2005) 80-84; https://doi.org/10.1055/s-2004-830536

23. L. S. Chupak, X. Zheng, S. Hu, Y. Huang, M. Ding, M. A. Lewis, R. S. Westphal, Y. Blat, A. McClure and R. G. Gentles, Structure activity relationship studies on chemically non-reactive glycine sulfonamide inhibitors of diacylglycerol lipase, Bioorg. Med. Chem. 24 (2016) 1455-1468; https://doi. org/10.1016/j.bmc.2016.02.006

24. F. J. Janssen, H. Deng, M. P. Baggelaar, M. Allarà, T. van der Wel, H. den Dulk, A. Ligresti, A. C. van Esbroeck, R. McGuire, V. Di Marzo, H. S. Overkleeft and M. van der Stelt, Discovery of glycine sulfonamides as dual inhibitors of sn-1-diacylglycerol lipase $\alpha$ and $\alpha / \beta$-hydrolase domain 6 , J. Med. Chem. 57 (2014) 6610-6622; https://doi.org/10.1021/jm500681z

25. J. Kim, Y. S. Lee, C. S. Kim and J. S. Kim, Betulinic acid has an inhibitory effect on pancreatic lipase and induces adipocyte lipolysis, Phytother. Res. 26 (2012) 1103-1106; https://doi.org/10.1002/ptr.3672

26. Y. Bustanji, I. M. Al-Masri, M. Mohammad, M. Hudaib, K. Tawaha, H. Tarazi and H. S. Alkhatib, Pancreatic lipase inhibition activity of trilactoneterpenes of Ginkgo biloba, J. Enzyme Inhib. Med. Chem. 26 (2011) 453-459; https://doi.org/10.3109/14756366.2010.525509

27. Y. M. Al-Hiari, V. N. Kasabri, A. K. Shakya, M. H. Alzweiri, F. U. Afifi, Y. K. Bustanji and I. M. AlMasri, Fluoroquinolones: novel class of gastrointestinal dietary lipid digestion and absorption inhibitors, Med. Chem. Res. 23 (2014) 3336-3346; https://doi.org/10.1007/s00044-014-0913-4

28. P. Hadváry, W. Sidler, W. Meister, W. Vetter and H. Wolfer, The lipase inhibitor tetrahydrolipstatin binds covalently to the putative active site serine of pancreatic lipase, J. Biol. Chem. 266 (1991) 2021-2027.

29. C. Schouand and N. H. Heegaard, Recent applications of affinity interactions in capillary electrophoresis, Electrophoresis 27 (2006) 44-59; https://doi.org/10.1002/elps.200500516

30. A. Lookene, N. Skottova and G. Olivecrona, Interactions of lipoprotein lipase with the active-site inhibitor tetrahydrolipstatin (Orlistat), Eur. J. Biochem. 222 (1994) 395-403; https://doi. org/10.1111/j.1432-1033.1994.tb18878.x

31. H. Lee, S. Cao, K. E. Hevener, L. Truong, J. L. Gatuz, K. Patel, A. K. Ghosh and M. E. Johnson, Synergistic inhibitor binding to the papain-like protease of human SARS corona virus: mechanistic and inhibitor design implications, Chem. Med. Chem. 8 (2013) 1361-1372; https://doi.org/ 10.1002/ cmdc. 201300134

32. C. W. Murray and T. L. Blundell, Structural biology in fragment-based drug design, Curr. Opin. Struct. Biol. 20 (2010) 497-507; https://doi.org/10.1016/j.sbi.2010.04.003 